\title{
PUBLIC TELEPHONE NETWORK VULNERABILITIES
}

\author{
G. Lorenz, J. Keller, G. Manes, J. Hale, S. Shenoi
}

\begin{abstract}
The convergence of public telephone networks (PTNs), wireless networks and the Internet makes it possible to disrupt vital information and communications systems from afar with software. PTN vulnerabilities are also growing due to system complexity, deregulation, increasing numbers of highly-skilled attackers, and the automation of attacks. This paper analyzes PTNs vulnerabilities, and presents an attack taxonomy. The taxonomy considers security threats posed by in-band attacks, out-of-band attacks, wireless attacks and Internet attacks.
\end{abstract}

Keywords: Public telephone networks, converged networks, vulnerabilities, attack taxonomy

\section{INTRODUCTION}

Public telephone network (PTN) fraud originated in 1961, when Blue Boxes were used to make free long-distance calls [4]. In the 1970s, "phone phreaking" drew attention: whistles packaged in Cap'n Crunch cereal boxes were used by "phreakers" to generate control tones, enabling them to modify telephone service at will [4]. The explosion of global communications has increased the scope and magnitude of fraud $[4,14,19]$. In 1997, a fifteen-year-old breached telephone systems at a high tech company to make free calls, access voice mail and eavesdrop on company business [4]. In 1998, a company lost $\$ 700,000$ in two days from PBX attacks [4]. Worldwide losses - due to toll fraud, insider misuse, cell phone cloning and slamming - now exceed $\$ 30$ billion a year [4].

But new dangers are posed by insidious penetrations into PTN networks $[19,22]$. Most penetrations are by thrill-seeking hackers and novices using "kiddie scripts." However, criminals, terrorists and foreign governments are also systematically probing PTNs, potentially monitoring communications and searching for vulnerabilities [22].

Public telephone networks are massive heterogeneous networks comprising a backbone, wireless networks and the Internet. The PTN backbone, driven by the Signaling System 7 (SS7) protocol suite $[1,2,18]$ has significant vulnerabilities $[10,17]$. Originally designed for a closed industry, SS7 incorporates weak authentication facilities. However, deregulation requires phone companies to 
provide SS7 connections to any entity [19]. Increased PTN access, coupled with Internet-PTN convergence (e.g., xDSL, Dial-Up and ISDN connections) $[8,23]$, make it easier for attackers to enter and then exploit poorly secured SS7 networks [10].

Wireless networks, an explosive growth area [6,9], also pose serious risks. Wireless communications are inherently vulnerable. Moreover, the rapid evolution of wireless technologies, the lack of standards and the pervasive use of legacy systems give attackers opportunities to wreak havoc on a massive scale $[10,19,22]$.

Accentuating the problem is the lack of sustained research and development efforts focused on PTN security [22]. Very few systems exist for securing PTNs $[20,21]$. Published research and prototypes for defending PTNs against attacks are virtually non-existent.

This paper focuses on the problem of securing PTNs. The PTN backbone, wireless networks and the Internet-PTN convergence are described in detail. Vulnerabilities are analyzed to obtain a taxonomy of attacks on PTNs. The taxonomy considers security threats posed by four separate PTN attack modes: in-band attacks, out-of-band attacks, wireless attacks and Internet attacks. The taxonomy provides an opportunity for the systematic development of mitigative and preventive strategies, and the identification and classification of emerging threats.

\section{PTN OVERVIEW}

Public telephone networks (PTNs) are massive heterogeneous networks comprising three overlapping sub-networks: a PTN backbone, the Internet and wireless networks (Figure 1). The PTN backbone primarily uses the SS7 protocol $[1,2,18]$, the Internet employs the TCP/IP prototol suite [5,12], and wireless networks use the CDMA, TDMA or GSM protocols $[6,9,16]$. The PTN backbone is connected to the Internet and wireless networks via IP and wireless gateways, respectively. In addition, the PTN backbone maintains various connections to entities ranging from individual users to large organizations. For example, individuals may use xDSL, Dial-Up and ISDN connections to access the PTN (and Internet). Large organizations employ analog trunks to connect priyate branch exchanges (PBXs) to the PTN.

Attackers have numerous points of entry due to network convergence $[8,19$, 23]. Indeed, every point of attachment between the sub-networks represents an opening to potential attackers.

The Internet (attack point 1 in Figure 1) is a convenient platform for launching attacks on the PTN. Numerous hacker exploits can be used to compromise or seize control of Internet hosts connected to PTN elements. The threats are increased as convergence technologies, e.g., xDSL, dial-up and ISDN, propa- 


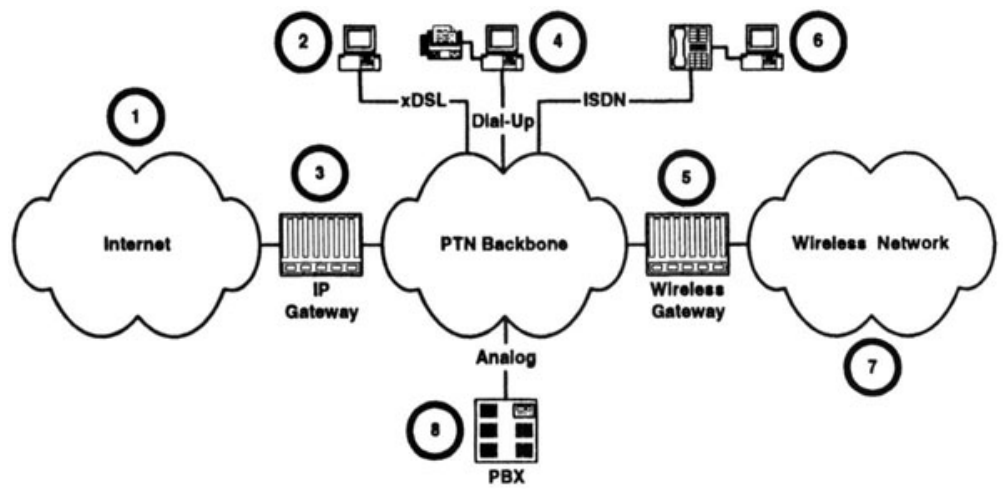

Figure 1. PTN overview (with attack points numbered).

gate (attack points 2,4 and 6). The concern is that while they provide increased access to PTN services, convergence technologies are intrinsically insecure.

PTN gateways (points 3 and 5) are highly vulnerable. These gateways authenticate users before providing access. However, attackers who circumvent the authentication procedures have free reign over PTN assets.

Wireless networks (attack point 7) open new vulnerabilities. Especially problematic are services providing wireless access to the Internet.

PBXs often use legacy technologies (in-band signaling) which are known to have serious vulnerabilities (colored box attacks [7,10,19]; attack point 8). Indeed, PBXs - because they use many connections to the PTN backbone - are often used as bounce points for attacks [17].

Thus, the PTN backbone is completely surrounded by expanding networks, each with significant vulnerabilities that provide attractive entry points for attackers. At the same time, the PTN backbone is the central and most important component of the global communications network. A significant portion of the Internet uses leased telephone lines and wireless communications are routinely routed through land lines. If the PTN backbone fails, everything else will fail.

\section{PTN BACKBONE VULNERABILITIES}

The U.S. PTN incorporates four regional Bell operating companies (RBOCs) (Verizon, SBC, BellSouth and Qwest) and numerous independent phone companies, all connected by long-distance providers. Figure 2 shows an RBOC supporting telephone customers as well as small companies (CLECs) that use X.25/SS7 data lines. Each RBOC is connected to other RBOCs via long distance companies (possibly themselves).

The PTN backbone uses signaling, transmission and operations equipment (Figure 2) [18]. Signaling equipment sets up and tears down calls, and includes databases and processors for number translation and call routing. Transmission 
equipment carries telephone conversations. Operations equipment, including the operations support system (OSS), performs management, inventory, engineering and repair functions.

\section{Signaling}

PTNs use in-band or out-of-band signaling. The older in-band technique combined signaling and transmission over copper wires, but it was inefficient and did not support advanced services [2]. Modern out-of-band communication between PTN central-office switches occurs via dedicated (private and public) X.25 data networks using the SS7 protocol for setting up and tearing down calls, establishing billing and returning busy signals [18]. These dedicated networks prevent in-band fraud and conserve resources. However, modern telecommunications systems still use in-band signaling because it is more easily deployed.

In-band signaling uses multi frequencies (MF) and dual tone multi frequencies (DTMFs) - tones generated using telephone touchpads - to send control signals [2]. MFs send control signals directly to telephone trunks and switches. DTMFs are used for number dialing, ringbacks, busy signals, dial tones; they also activate and deactivate services, e.g., call-forwarding (*72/*73) and callreturn $(* 66 / * 69)$.

Out-of-band signaling using SS7 has largely replaced in-band DTMF for the U.S. PTN $[2,19]$. An international standard, SS7 governs network architecture and configuration, and message transport $[1,18]$. The architecture includes: (i) service switching points (SSPs), the switches that handle base communications; (ii) signal transfer points (STPs), the switches that handle routing; and (iii) service control points (SCPs) that provide database access for routing and advanced PTN services, e.g., caller ID, call forwarding and toll-free calls. To increase reliability, SS7 elements are deployed in redundant mated pairs. The SS7 protocol reflects the OSI model, splitting the message transport layer (MTP) into three levels (MTP 1-3: the physical, data link and network equivalents to the OSI), and call management (ISUP, TUP) and advanced services (SCCP, TCAP), which are built on top of the MTP layer $[1,18]$.

\section{Transmission}

The U.S. PTN was originally designed to carry analog signals. Currently, transmission initiates with analog signals being sent to a central office where they are converted to digital to facilitate switching and routing within the PTN [1]. Digital signals are converted back to analog via pulse code modulation (PCM) sampling before their presentation at the destination. Using time division multiplexing (TDM), the PCM is transmitted over a physical connection for the call duration. Voice transmission typically employs channelized $\mathrm{T} 1$ (1.544Mbps) circuits. (Each T1 line has 24 64kbps trunk circuits over two copper pairs; each call uses one circuit.) Synchronous TDM is inefficient be- 


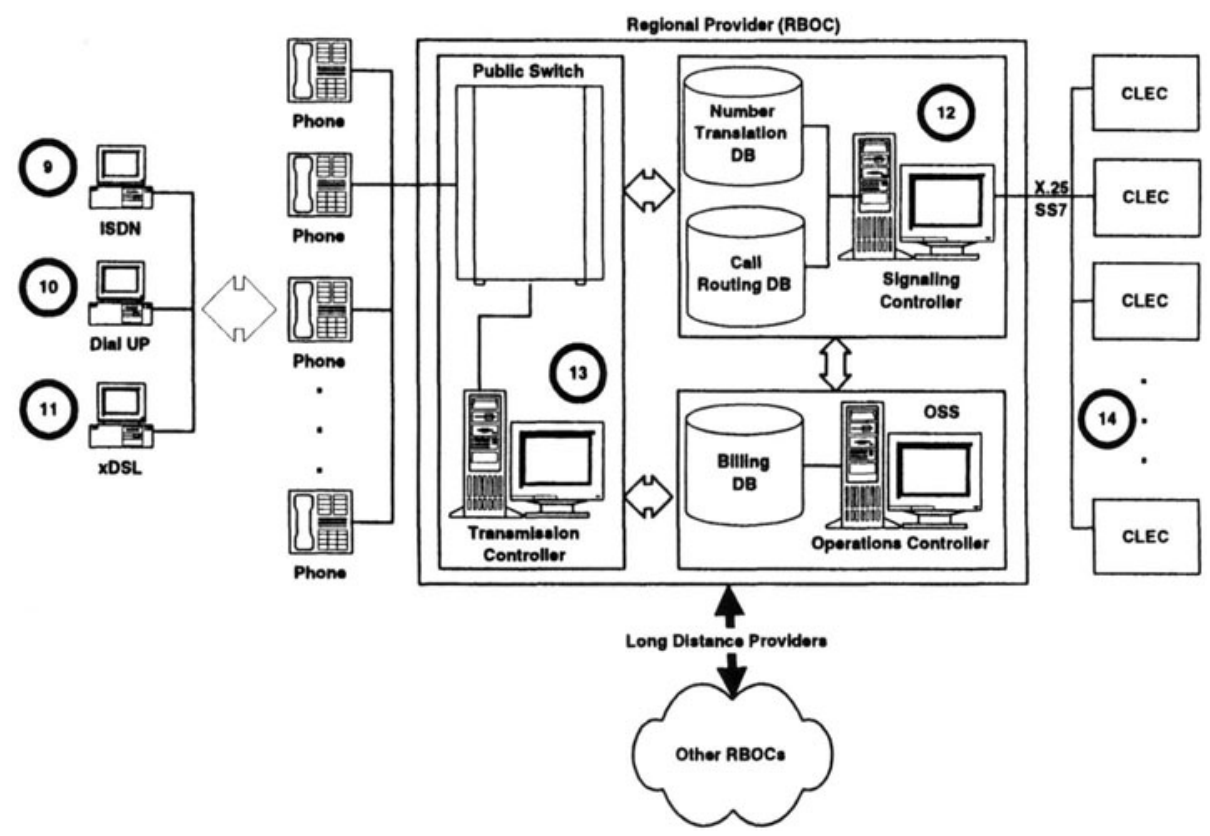

Figure 2. PTN backbone (with attack points numbered).

cause each time slot is dedicated to the user for the call duration, even when nothing is transmitted. Modern transmission systems address this inefficiency by integrating heterogeneous technologies, e.g., voice over IP (VoIP).

\section{Operations}

Operations support systems (OSSs) are the remote maintenance centers of PTNs $[1,18,19]$. They perform management, inventory, engineering, planning and repair functions. Originally, OSSs were mainframe systems that supported business operations. Modern OSSs support these operations, but also manage network systems and advanced customer services. OSSs comprise several computer systems and databases (service control points (SCPs) described above) that handle ordering, inventory, activation and customer information services throughout PTNs via digital interfaces. The systems are also responsible for updating, maintaining and monitoring all SCP databases. A service management system (SMS) consisting of a command set and several GUIs provides a standard interface to SCP databases. SMS also maintains SCP integrity: it acts as a central point to update SCP databases, which then propagate throughout the SS7 backbone.

\section{Vulnerabilities}

The PTN backbone is susceptible to numerous threats. The replacement of in-band signaling with out-of-band (SS7) signaling largely eliminated the well-known colored box attacks $[1,7,10,19]$. However, the SS7 architecture and 
protocol are vulnerable because they possess limited authentication mechanisms (SS7 was originally designed for a closed community) $[17,18,19]$. Moreover, deregulation coupled with the proliferation of VoIP and other integrated services (attack points 9,10 and 11 in Figure 2) significantly increase the vulnerability to fraud, eavesdropping and denial of service attacks $[14,19,22]$.

Major PTN vulnerabilities stem from the SS7 signaling system [19]; thus, SS7 components (points 12 and 14) are prone to numerous exploits. PTN databases or service control points (SCPs) - such as number translation, call routing and billing databases - are particularly vulnerable. Attackers can wreak havoc by modifying important information maintained in these databases. During a terrorist bombing incident, an attacker can modify entries in a call forwarding database to re-route all phone calls to emergency services (e.g., 911), disrupting them and increasing the number of casualties.

Transmission systems, especially central office switches, are also prime targets (attack point 13). These switches are vital to PTN operation, but they are vulnerable to a range of denial of service attacks.

\section{WIRELESS NETWORK VULNERABILITIES}

Wireless networks are the largest growth sector for voice communications, and are used increasingly for data transmission [6]. A typical network is shown in Figure 3. It comprises a cellular system linked to a base station subsystem via tower/transceivers. The base station subsystem is connected to a network subsystem and ultimately to the PTN backbone using SS7 (X.25) data lines.

The cellular system is a network of towers that define cells. Radio signals received by a tower in the originating cell are routed to the corresponding base station subsystem. This subsystem comprises a base transceiver station (BTS) and a base station controller (BSC). The BTS is responsible for radio transmission and reception in one or more cells within a service area. It contains radio transceivers that define cells and handle the radio-link protocols with handsets (mobile stations). The BSC manages radio resources for one or more BTSs, handling radio-channel setup, frequency hopping and handovers. It also connects the mobile stations to the network subsytem.

Wireless network subsystems are essentially "mini" PTN regional providers (RBOCs), i.e., they use SS7 signaling, circuit switching and operations support systems. As shown in Figure 3, a wireless network subsystem comprises a mobile switching center (MSC), equipment identity register (EIR), authentication center (AuC), home location register (HLR), and a visitor location register (VLR). The MSC provides circuit switched network access, database access and connections to the PTN backbone. The MSC, in conjunction with other network subsystem entities, also handles registration, authentication, location updating, handovers and call routing to mobile subscribers. 


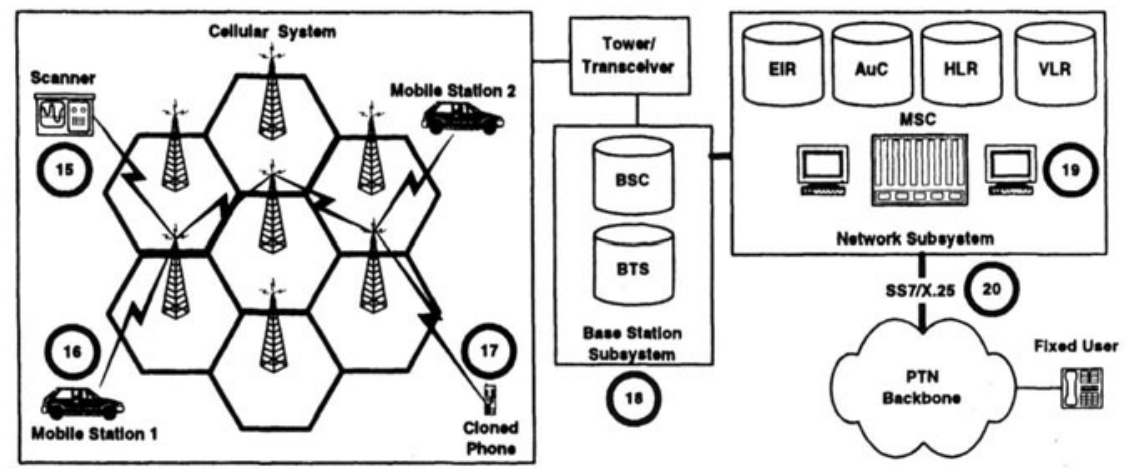

Figure 3. Typical wireless network (with attack points numbered).

The EIR database contains lists of valid mobile devices, allowing providers to deny service and track stolen/cloned equipment. The AuC database stores secret keys corresponding to subscriber identification modules (SIMs) and electronic serial numbers (ESNs) used for authentication and encryption. HLR and VLR (and MSC), provide call-routing and roaming capabilities. HLR and VLR contain all administrative information pertaining to registered subscribers and their locations. VLR works in conjunction with HLR to locate roaming users. When a mobile station enters a new network cell, data are entered into the VLR so that it can be located. Wireless networks provide excellent mobility, but they are very complex and are not yet standardized.

Wireless communication has inherent risks because radio signals are readily intercepted and exploited [16]. Eavesdropping is widespread. All that is needed is a scanner tuned to the right frequency (point 15).

Phreakers perform cloning $[4,16]$ by stealing the security codes (e.g., electronic serial numbers (ESNs)) of legitimate mobile stations (Figure 3). Typically, ESNs are gleaned using scanning devices. Cellular phones are reprogrammed with stolen ESNs to create cloned phones (point 17). A cellular cache box automates this process [7]. It captures an ESN, uses it once, purges it from memory, and then repeats the cycle. Some protocols, e.g., CDMA, scramble data using psuedo-random noise codes, effectively eliminating scanner-based eavesdropping and cloning [16]. However, this technology is limited and expensive. Meanwhile, phreaking techniques are continuously improving, e.g., using frequency-hopping scanners [7].

Denial of service attacks are increasingly directed at wireless networks. Attackers masquerading as legitimate mobile stations have flooded radio towers, denying service to thousands of users (point 16) $[4,19]$. The Timofonica virus of June $\mathbf{2 0 0 0}$ was designed to flood cell phones that had text capabilities, posing a serious threat to millions of handheld computers, pagers and phones [3]. 


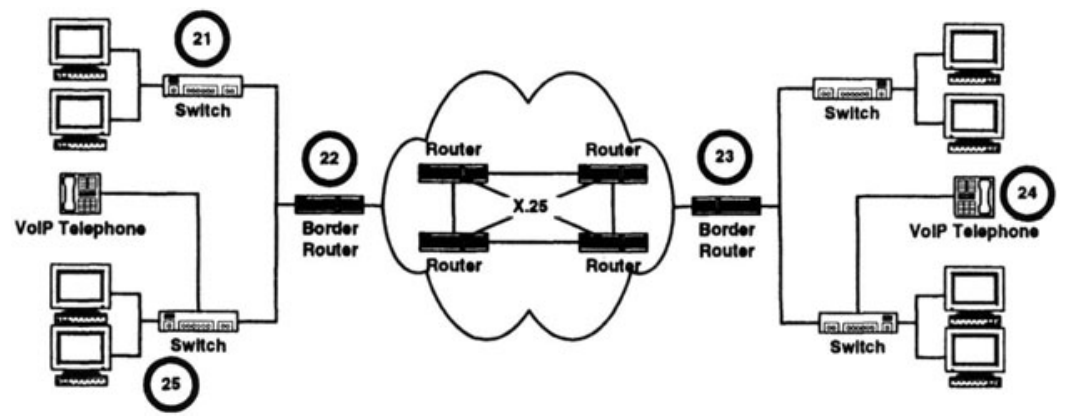

Figure 4. Internet backbone (with attack points numbered).

Radio signals received by a cellular tower are routed to a base station subsystem before being sent to the network subsystem (Figure 3). The base station subsystem thus interfaces radio signals to the hard line. It is an attractive target (point 18) because it is a single point of failure for an entire sector of wireless cells.

Wireless network subsystems, like RBOCs in the PTN backbone, use the SS7 architecture and protocol. Thus, the vulnerabilities described in Section 3 apply here (point 19). Specialized databases are also vulnerable. For example, each sector of cells maintains VLR and HLR databases with the ESNs of all mobile stations in the proximity. Attackers can tap into these databases and steal ESNs. Conversely, they can perpetrate large-scale denial of service attacks by modifying, erasing or shutting down these vital databases.

Cellular networks rely on connections to the PTN backbone. Attackers can seize control of a wireless network subsystem and then utilize its PTN connections to launch a slew of attacks (point 20).

\section{INTERNET VULNERABILITIES}

Originally developed for data transmission, the Internet is increasingly carrying voice transmissions [5]. The Internet backbone is primarily a collection of routers linked by X.25 data lines leased from PTNs (Figure 4). Individual networks use border routers to connect to this backbone.

Internet (VoIP) telephony is more efficient than PTN voice transmission (using TDM), but the quality of service (QoS) is not as good. Nevertheless, the attraction of free long-distance has significantly increased VoIP applications, blurring the PTN-Internet distinction.

The increasing interdependence and interconnectivity between the Internet and the PTN backbone makes Internet vulnerabilities (e.g., CVE and ICAT databases [11,13]) particularly relevant $[19,22]$. Large portions of the Internet use leased phone lines. Conversely, PTN components are networked using Internet technologies, and often, the Internet itself. Thus, Internet vulnerabilities affect the PTN backbone. 
Packet sniffing is a major concern. Sniffers attached to Internet switching devices can intercept email, e-commerce transactions, as well as user names and passwords passed as plaintext (attack point 21). VoIP telephony enables attackers to eavesdrop on voice communications as they traverse the Internet (point 24). Furthermore, attackers who compromise Internet routers can direct traffic to compromised switches (points 22 and 23). Such routing attacks give sniffers access to much broader areas, particularly if core routers (point 23) are compromised.

Internet vulnerabilities can be exploited from any host (point 25). Therefore, attacks can be launched remotely at any system within the PTN backbone, within wireless networks or within the Internet itself. Note that since Internet hosts are routinely infiltrated, they will become the primary attack points in the converged PTN-Internet environment.

\section{PTN ATTACK TAXONOMY}

A PTN attack taxonomy provides an opportunity for the systematic development of mitigative and preventive strategies, and the identification and classification of emerging threats. The taxonomy presented in Figure 5 and discussed in this section considers the four main security threats: modification, interception, interruption and fabrication [15]. Four separate attack modes are examined: in-band attacks, out-of-band attacks, wireless attacks and Internet attacks.

\subsection{PTN In-Band Attacks}

In-band (colored box) attacks have been largely eliminated by SS7-based out-of-band signaling [18]. However, private branch exchanges (PBXs) used by large enterprises commonly rely on in-band signaling, and are therefore vulnerable to colored box attacks [10]. Moreover, certain advanced services engage in-band signaling [2]. E.g., caller ID requires that the caller's telephone number and the date/time of the call be sent in ASCII format within the first 4-second silent interval in the ringing cycle. This protocol can be easily spoofed or disrupted.

In-band modification attacks mainly involved unauthorized alteration of central office switches to commit toll fraud. Modification attacks, and the three other attack types, were also perpetrated by tampering with switches using colored boxes $[7,10]$, e.g., a chartreuse box that facilitated the theft of electricity from phone lines [7].

Interception was historically performed using wire taps. However, it was simplified by special colored boxes, e.g., brown and dayglo, designed for eavesdropping [7]. In addition, copper and blotto boxes facilitated sabotage by enabling service interruption without the need to physically cut lines [7]. 


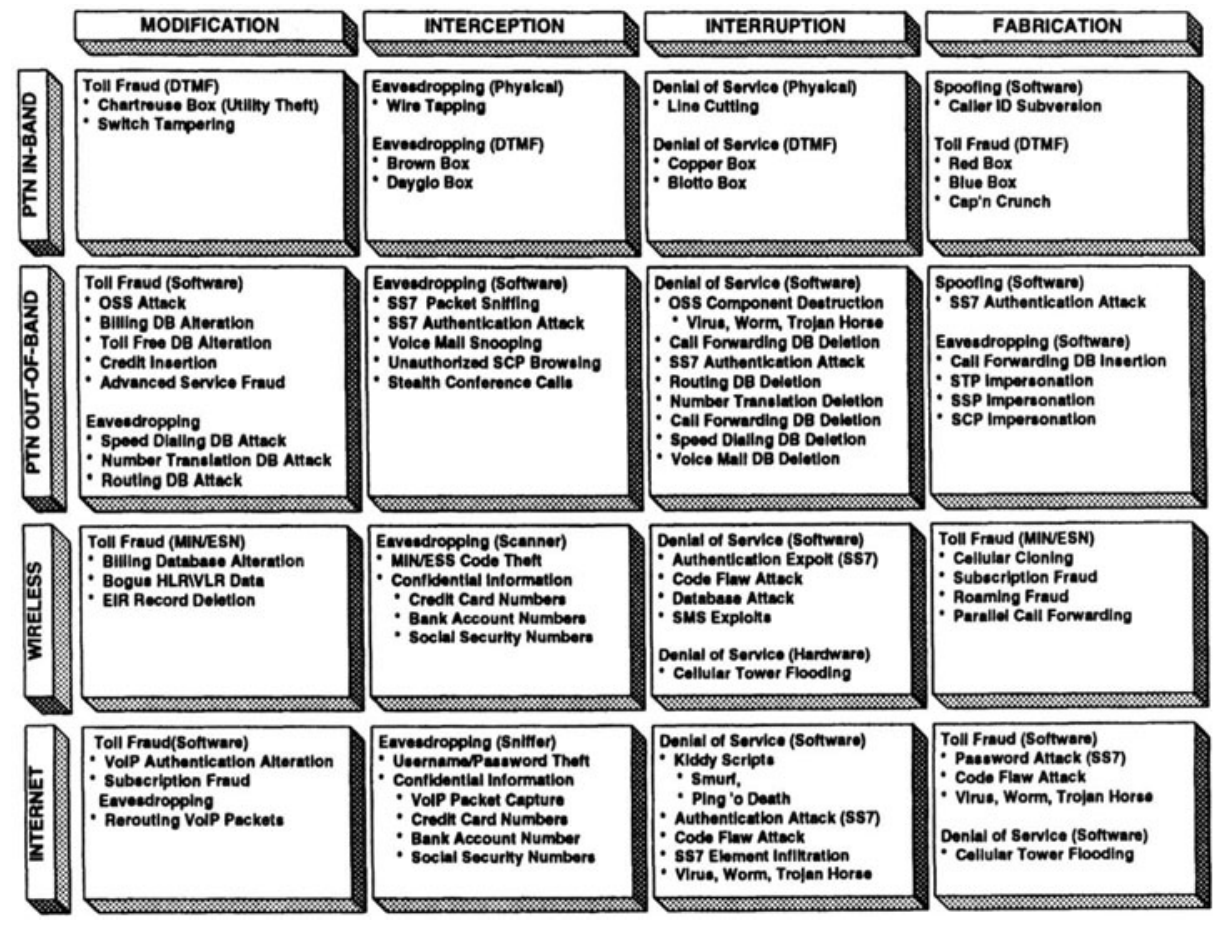

Figure 5. PTN attack taxonomy.

Colored box attacks utilized fabricated tones to gain unauthorized access to PTN components $[4,7]$. For example, a red box simulated coin (quarter) tones required by the automated coin toll system (ACTS), permitting free calls from pay phones. The legendary "Cap'n Crunch" whistles and blue boxes produced $2600 \mathrm{~Hz}$ tones for seizing control of switches [4]. In fact, a veritable rainbow of colored boxes [7] was available for in-band attacks (Figure 6).

\subsection{PTN Out-of-Band Attacks}

Out-of-band attacks primarily abuse the SS7 protocol. SS7 was designed for a closed industry and, therefore, incorporates weak authentication facilities $[18,19]$. However, deregulation now requires phone companies to provide SS7 connections to any entity for a $\$ 10,000$ fee [19]. This, coupled with the InternetPTN convergence, makes it easier for attackers to enter and then exploit poorly secured SS7 networks.

Once entry is gained, attackers could target network databases with critical information. For example, call forwarding, speed dialing, number translation or routing databases can be modified to re-route calls and intercept or interrupt transmissions. Attackers could also erase billing records, modify toll free num- 
- Black - Allows the calling party not to be billed for calls

- Blue - Emulates an operator by seizing a trunk with a $2600 \mathrm{hz}$ tone

- Brown - Creates a party line from two phone lines

- Chartreuse - Uses the electricity from a phone line

- Dark - Re-routes outgoing or incoming calls to another phone

- Green - Emulates the coin collect, coin return, and callback (DTMF) tones

- Pearl - Generates tones

- Red - Generates quarter tones allowing free phone calls from pay phones

- Silver - Generates tones for ABCD keys

- Violet - Keeps a payphone from hanging up

- White - Acts as a portable DTMF keypad

Figure 6. Common PTN colored-boxes.

bers, insert credit and update subscribed services records. They could obtain unlimited free calls, as well as call waiting, caller ID and conference calling, by simply altering the appropriate customer records.

Interception can be accomplished in numerous ways, especially by voice mail snooping and SS7 packet sniffing. SS7 communications are transmitted in plaintext. This enables attackers to obtain confidential user information without having to access PTN databases (SCPs).

PTN interruptions can be accomplished by deleting data maintained by SS7 network components (particularly SCPs). Data deletion can completely disrupt a network, yet this is very easy to do once access is gained to an SS7 network, especially its operations support system (OSS). The deletion of records from call forwarding, speed dialing and voice mail databases produces relatively minor service interruptions. However, deleting information maintained in routing and number translation databases can cause large-scale outages.

PTN computer systems are vulnerable to trojans, viruses and worms. Also, fabricating credentials - or spoofing - is facilitated by weak SS7 authentication and expanding network connections. Attackers masquerading as legitimate SS7 entities can wreak havoc throughout the PTN.

\subsection{Wireless Attacks}

Wireless communications are most vulnerable to toll fraud, eavesdropping and denial of service. Attacks are relatively easy because wireless technology is inherently insecure $[3,16]$.

Unauthorized modification of wireless network databases is a serious threat to wireless communications and to the operation of the PTN backbone. E.g., inserting bogus home location register (HLR) and visitor location register (VLR) data allows attackers to activate and use phones free of charge. Such falsified data allows attackers to fool networks into believing that fraudulent wireless 
devices are legitimate. Since wireless phone calls eventually travel through the PTN backbone, this type of fraud affects the entire PTN. Likewise, modification attacks could target equipment identity register (EIR) databases to increase the fraudulent use of illegal or cloned equipment.

Interception attacks are facilitated by the nature of wireless transmissions and the availability of inexpensive scanners. Attackers have been known to scan transmissions and steal electronic serial numbers (ESNs) to create cloned wireless devices. In addition, sensitive information, e.g., credit cards, social security numbers and bank account information, can be intercepted by scanning wireless communications.

Wireless interruptions can be accomplished in a variety of ways. Since databases are vital to call processing, denying access to them cripples a network. E.g., slowing or interrupting HLR and VLR database accesses will stifle call processing as call routing cannot proceed. A denial of service attack could be launched by broadcasting numerous short message service (SMS) messages. Alternatively, a brute force attack could simply flood reserved wireless transmission frequencies.

Fabrication attacks (cellular cloning) are a major concern for wireless communications providers. Indeed, this type of fraud costs providers several billion dollars a year [4].

\subsection{Internet Attacks}

Internet attacks are well documented [11,13]. The focus here is on attacks that can affect PTN systems and operations.

Modification attacks are typically aimed at VoIP toll fraud and eavesdropping. E.g., altering VoIP authentication data allows attackers to circumvent billing and call verification. Likewise, modifying Internet routing tables (by compromising routers) gives attackers the ability to re-route and intercept VoIP communications.

Interception, e.g., eavesdropping on conversations and data, is simplified by IP packet sniffers. In fact, the proliferation of sophisticated sniffing software is a huge concern as the Internet and PTNs converge.

Interruption attacks, like those regularly perpetrated on the Internet, will become increasingly common. Given that networked computers control most PTN services, hackers and script kiddies can launch significant denial of service attacks. Attackers could also deploy viruses and trojans to target key PTN components, creating widespread service outages.

Internet-based fabrication attacks will be significant in converged environments. E.g., attackers could use spoofing software to bypass authentication procedures, gain unauthorized access to PTN components, and perpetrate attacks ranging from toll fraud to service disruption. 


\section{CONCLUSIONS}

PTN vulnerabilities are growing due to system complexity, deregulation, increasing numbers of highly-skilled attackers, and the automation of attacks. Unfortunately, relatively little research has specifically addressed PTN vulnerabilities.

The attack taxonomy described in this paper considers in-band attacks, outof-band attacks, wireless attacks and Internet attacks. It provides an opportunity for the systematic development of mitigative and preventive strategies, and the identification and classification of emerging threats. We hope that this work will stimulate further studies of PTN vulnerabilities and the development of sophisticated systems for safeguarding vital telecommunications networks

\section{References}

[1] Black, U. (1997) ISDN and SS7: Architectures for Digital Signaling Networks. Prentice Hall, Upper Saddle River, New Jersey.

[2] Bosse, J. (1997) Signaling in Telecommunication Networks. Wiley, New York.

[3] Cable News Network (June 12, 2000) Palm antivirus protection in the works (www.cnn.com).

[4] Denning, D. (1999) Information Warfare and Security. Addison-Wesley, Reading, Massachusetts.

[5] Faynberg, I. et al. (2000) Converged Networks and Services: Internetworking IP and the PSTN. Wiley, New York.

[6] Glisic, S. et al. (1997) Wireless Communications: TDMA v.CDMA. Kluwer, Boston.

[7] Hacker Quarterly (2001) (www.2600.com).

[8] Kuhn, P. et al. (1999) Broadband Communications: Convergence of Network Technologies. Kluwer, Boston, Massachusetts.

[9] Lin, Y. et al. (2000) Wireless and Mobile Network Architectures. Wiley, New York.

[10] Lorenz, G. et al. (2000) Characterization of attacks on public telephone networks, Proceedings of the SPIE Conference on Technology on Law Enforcement. Boston, Massachusetts.

[11] MITRE Corporation (2001) Common Vulnerabilities and Exposures (CVE) (cve.mitre.org).

[12] Muller, N. (1999) IP Convergence: The Next Revolution in Telecommunications. Artech, Norwood, Massachusetts.

[13] National Institute of Standards and Technology (2001) I-CAT Database (csrc.nist.gov/icat).

[14] Network Reliability and Interoperability Council (1997) Final Report of the Network Reliability and Interoperability Council. Federal Communications Commission, Washington, D.C.

[15] Pfleeger, C. (1997) Security in Computing. Prentice Hall, Upper Saddle River, New Jersey.

[16] Rhee, M. et al. (1998) CDMA Cellular Mobile Communications and Network Security. Prentice Hall, Upper Saddle River, New Jersey.

[17] Rozenblit, M. (2000) Security for Network Telecommunications Management. IEEE Press, Piscataway, New Jersey.

[18] Russell, T. (2000) Signaling System \#7. McGraw-Hill, New York. 
[19] Schneider F. (Ed.) (1999) Trust in Cyberspace. National Academy Press, Washington, D.C. [20] SecureLogix Corporation (2001) San Antonio, Texas (www.securelogix.com).

[21] Sevis Systems (2001) San Antonio, Texas (www.sevis.com).

[22] Wadlow, T. (2000) The Process of Network Security. Addison-Wesley, New York.

[23] The White House (2000) Defending America's Cyberspace: National Plan for Information Systems Protection, Washington, D.C. 